\title{
Praktische Auswirkungen der Dienstleistungsfreiheit auf die Versicherung in der EG
}

\author{
von Prof. Dr. jur. Dr.-Ing. E. h. Reimer Schmidt*
}

1. Dieser Vortrag stellt keinen Beitrag zur Rechtsetzung der EG dar. Ich spreche nicht de lege ferenda, sondern darüber, wie die Praxis etwa mit dem, was auf diesem Gebiet bisher geschaffen oder vorgeschlagen worden ist, fertig werden wird. Der Vortrag löst sich zudem von dem Interessenwiderstreit, der mit jedem Rechtsetzungsverfahren verbunden ist, und soll zugleich auch der unzutreffenden Vorstellung entgegenwirken, das Versicherungsgewerbe in den Mitgliedstaaten dächte nicht über die praktischen Probleme des Binnenmarktes nach, eines Binnenmarktes, den es nicht erst 1992 geben wird.

Ich frage mich zudem, ob nicht über Versicherung zur Zeit zu viel „von höherer Warte" aus gesprochen wird. Schon immer hat der Umstand, daß es sich um Dienste höherer Art handelt, die „invisible“ sind, zu vielfältigen Analysen und Erklärungen von Gegenstand und Funktion der Versicherung geführt. Die Diskussionsdichte ist aber in jüngster Zeit sehr groß geworden, wenn man an den Ruf von jenseits des Kanals denkt, daß die (eigene) Assekuranz erwachen möge, und an die vielfältigen Überlegungen zum Thema „1990 als Zieljahr der Assekuranz”, um eine headline der NZZ zu nennen. Es kommt hinzu, daß durch die Rechtsetzungstechnik der EG in Richtlinien sozusagen „Jahresringe” der Gesetzgebung entstehen. Die Rechtsetzungsorgane der EG und der Mitgliedsländer diese bei der Umsetzung in die nationalen Rechte - müssen sich immer wieder, gleichsam jährlich, mit der Materie beschäftigen, haben immer neue - teilweise auch doktrinäre - Gedanken und stellen das Rechtsgefühl des Volkes vor eine schwere Probe und den Rechtsbefolgungswillen der Marktteilnehmer bisweilen in Frage. Dabei ist die Rechtsbefolgungsintensität in den Mitgliedstaaten ohnehin unterschiedlich. Objektive Tätigkeitsmöglichkeiten in anderen Ländern und subjektive Beurteilungsschemata werden innerhalb der Mitgliedsländer der EG und außerhalb mit Recht und notwendig diskutiert: der Stimulierungseffekt, den Liberalisierung nun einmal hat, hat einen Aktionismus auch der Worte ausgelöst. In diesem Zusammenhang löst der insbesondere in den USA entwickelte Gedanke der Deregulierung recht weitreichende Folgerungen aus. Die Deregulierung regt, wie eben erwähnt, die Eigeninitiative der Wirtschaftenden an, indem sie den teilweise wild gewachsenen, teilweise aber auch auf jahrhundertealter Erfahrung beruhende Sockel der staatlichen Schutzvorschriften i.w. S. abbaut. Deregulierung steht deshalb in nicht unerheblichem Umfang in einem Spannungsverhältnis zu dem Konsumentenschutzgedanken. Jedenfalls wird sofort dann ein Re-Regulierungsbedürfnis sichtbar, wenn im Bereich der abgebauten

\footnotetext{
* Präsident der Genfer Vereinigung. Die Form des Vortrags ist beibehalten. Vorabdruck in „Versicherungswirtschaft", Karlsruhe 1989, Heft 1, S. 28-38.
} 
Schutzvorschriften technischer, medizinischer, finanzieller oder rein rechtlicher Art Schäden drohen oder eingetreten sind. Um dem vorzubeugen, geht häufig mit dem Abbau des vielleicht gröberen Netzes von Schutzvorschriften das Weben eines zwar feineren, aber dichteren Vorschriftensystems einher.

Zur Einführung darf ich noch eine Bemerkung machen: In dem gesamten Vortrag wird an die „völkerrechtlichen Nachbarschaften” (Schweiz, Österreich, Ostblockstaaten) ebenso gedacht wie an überdeckende völkerrechtliche Verträge, insbes. an GATT, UNO/ UNCTAD und auch (bezüglich der Bundesrepublik) USA (und an das besondere Verhältnis zum anderen deutschen Staat). Kurzum, die Analyse und Differenzierung der Rückwirkungen ist für uns (fast) ebenso wichtig wie für die genannten Staaten.

Die Überlegungen, die ich heute vortragen darf, werden von der eigenen Kritik voll erfaßt, weil es sich bei der Privatversicherung nun keineswegs um eine verschlafene Branche handelt; nur paßt ihr äußeres Bild nicht voll in die Vorstellungen vom Marktgeschehen, die in der theoretischen Nationalökonomie von sehr vielen Wettbewerbstheoretikern unter Zurückdrängung des Ordo-Gedankens besonders in letzter Zeit entwickelt worden sind. Es liegt mir daher daran, in diesem Vortrag vor allem die praktischen Perspektiven herauszuarbeiten.

Nach mehr als 33 Jahren Erst- und Rückversicherungspraxis spreche ich übrigens weder für die Verbände noch für Teilmärkte. Und ich hoffe, daß wissenschaftliche Schulung „trotz allem” meinen praktischen Blick nicht zu sehr getrübt hat.

Bevor ich mich der natürlich kritischen Reaktion auf dasjenige hingebe, was als Gemeinsamer Versicherungsmarkt auf die Mitgliedsländer zukommen mag, muß ich zunächst sagen, daß der Vertrag von Rom durch einen grandiosen Liberalisierungseffekt im gedanklichen Anschluß an das sog. „deutsche Wirtschaftswunder” die Eigeninitiative in den Teilwirtschaften der Mitgliedsländer in großartiger Weise mobilisiert hat.

Es ist heute interessant, daß die sechs Gründungsmitglieder seinerzeit im Rahmen der allgemeinen Vertragsziele des EWG-Vertrages, also über die Zollunion, die Harmonisierung und die Liberalisierung hinaus, jeweils bedeutende, ganz konkrete Ziele mit dem Beitritt zur Wirtschaftsunion verfolgten: Den Franzosen lag es brennend daran, die eigene Landwirtschaft zufriedenzustellen. - Ich brauche hier nicht zu behandeln, ob und ggf. inwieweit das uns noch heute bedrückende System vertragskonform war und ist. Die Deutschen dachten primär an einen freien Markt für Industrieerzeugnisse; die Italiener hatten vor allem den später so ungewöhnlich erfolgreichen „Export” von Arbeitskräften im Auge. Die drei kleineren Partner der ersten EG-Periode - Niederlande, Belgien, Luxemburg fühlten sich als Vehikel der Handels- und Finanzpolitik der Gemeinschaft.

Die Beitritte der (gleichfalls sechs) anderen Mitglieder sind sicherlich aus der Perspektive der Gemeinschaft als „Raketenstufe” der Liberalisierung gedacht, sie wirkten aber doch wohl nur teilweise so, wenn auch der Beitritt Großbritanniens für dessen Schwerpunkt Banking, Shipping, Airtraffic und Insurance bedeutsame neue Akzente setzte. Der Beitritt Spaniens relativ kurz nach dem Ende der Diktatur hat vorher kaum für möglich gehaltene wirtschaftliche Stimulationen gebracht. Auf die „Verwässerungsproblematik”, die bei Beitritt weiterer Staaten aufgeworfen sein könnte, kann ich hier nur hinweisen. Ich fühle mich aber deshalb zu einer kurzen, im Grunde positiven Zwischenbemerkung verpflichtet, weil Bilder ohne Hintergrund häufig der Perspektive ermangeln. 
2. Es kann und soll daher nichts über Tatbestände, Bedeutung und Funktion der Privatversicherung gesagt werden. Die Überlegungen stehen ganz unter praktischen Zielsetzungen in einer Situation, die durch die Initialzündung der Liberalisierung und Deregulierung der Finanzdienstleistungen gekennzeichnet ist. Allerdings kann ich einen theoretischen Gedanken nicht unterdrücken: Die Niederlassungsfreiheit geht von dem stimulierenden Effekt aus, den die Freiheit der Wahl des Produktionsortes auslöst. Die Dienstleistungsfreiheit soll ermöglichen, die Standortvorteile der Produktion gegenüber den Kunden anderer Bereiche zu nutzen. In beiden Fällen sollen die in den rechtlichen Rahmenbedingungen liegenden Handelshemmnisse durch Rechtsharmonisierung möglichst weitgehend abgebaut werden. Weil aber die Wettbewerber die Standortbedingungen, vor allem diejenigen des Steuerrechts und des Sozialrechts nur in geringem Maße beeinflussen können, sind der Optimierung hier relativ enge Schranken gesetzt. Der These, daß der Markt die Anpassung der Steuersätze in Kürze erzwingen werde, würde ich gern folgen, vermag es aber nicht. Die nationalen Gesetzgeber sind kaum bereit, die Standortnachteile für die eigenen Unternehmen unter Inkaufnahme von budgetären und sozialrechtlichen Nachteilen nennenswert zu mindern, so daß der Automatismus des Modells in der Praxis wesentlich größere Schwierigkeiten macht als in der Theorie. In diesem Zusammenhang werden die Harmonisierungsbemühungen der EG-Kommission allerdings nicht verkannt. Doch sind - bei aller Bejahung der Ziele des EWG-Vertrags - der Harmonisierung Grenzen gesetzt, vor allem durch die unterschiedlichen Rechtskulturen, das verschieden ausgeprägte Rechtsgefühl und den differenzierten Rechtsbefolgungswillen in den Mitgliedstaaten.

Betritt man das Feld des praktischen Versicherungsgeschäfts, so steht zunächst fest, $\mathrm{da} ß$ die Beseitigung der Beschränkungen der Niederlassungsfreiheit grundsätzlich in einer den Gegebenheiten des Versicherungsgeschäfts entsprechenden Weise erfolgt ist und zu einer stärkeren Integration der Märkte geführt hat. Dadurch, daß die Niederlassungen dem Recht des Tätigkeitslandes unterliegen - eine Regel, die bekanntlich der EuGH für das EG-Kartellrecht teilweise durchbrochen hat -, wurde den psychologischen Gegebenheiten des Massengeschäfts Rechnung getragen. Sie werden am deutlichsten in dem Werbeslogan „Der Vertreter wohnt nebenan”. Die Marktgewohnheiten wurden aber durch die den Versicherungsunternehmen eingeräumte Freiheit, sich niederzulassen, auch nicht grundsätzlich „europäisch verändert”. Es gibt vielleicht noch dies und das im Regelungsbild der Niederlassungsfreiheit zu ändern, der Aktivität der Gesetzesmacher sind hier aber doch Grenzen gesetzt. Es ist Wirksames geschehen.

Ich sollte die Fragen, die bezüglich der Auswirkungen der EG-Regelungen auf NichtMitgliedsländer auftreten, heute nicht besserwisserisch beurteilen, vor allem enthalte ich mich wirtschaftspolitischer Stellungnahmen. Immerhin gibt es noch die OECD, und im Felde des GATT tut sich einiges zu den Dienstleistungen, hoffentlich Gutes. Nach meiner Auffassung haben auf eigenständiger nationaler Gesetzgebung beruhende Lösungen der Nicht-Mitgliedsländer grundsätzlich den Vorrang vor Rechtsetzung durch oder auf Grund von völkerrechtlichen Verträgen. In diese Richtung scheint bisher auch die überwiegende öffentliche Meinung in anderen Nicht-Mitgliedsländern zu gehen, z. B. in Norwegen, Finnland und der Türkei. Ich meine, daß die Anpassung der eigenen Gesetzgebung (im Rahmen des Möglichen und Gewollten), also die gewillkürte Adaption deshalb wirksamer ist, weil die förmliche Übernahme des Rechtes des Gemeinsamen Marktes auf einem bestimmten Gebiet in Anbetracht der dauernden laufenden Weiterentwicklung immer nur eine „Momentaufnahme" darstellt und daß weitere förmliche Übernahmen eine automatische 
Adaption neue große Schwierigkeiten machen. Allerdings kommt man in diesem Falle nicht um das Reziprozitätsproblem herum: politische Lösungen könnten vielleicht dahin gehen, eine im Hinblick auf die Größen- und Leistungsverhältnisse des eigenen Binnenmarkts zum EG-Markt begrenzte und teilweise mehr symbolische Reziprozität zu gewähren. Die laufenden Berichte der Neuen Zürcher Zeitung zum Themenkomplex Schweiz und EG sind in diesem Zusammenhang als besonders wesentlich zu vermerken.

Allerdings blieben die selbständigen Tochtergesellschaften EG-ausländischer Mütter aus der bisherigen Regelung ganz heraus, so da $B$ nicht auf Versicherungsaufsichtsrecht beruhende Erschwernisse noch eine Rolle spielen können. Als „quartum comparationis” zusätzlich zur Niederlassung, Dienstleistung und Tochtergesellschaft wird jetzt die Europäische Aktiengesellschaft (der zweiten ,geistigen Auflage") diskutiert, die nach EG-Recht lebt und deren Niederlassungen im EG-Ausland und deren dortige Dienstleistungen wie solche der Tätigkeitsland-Gesellschaften behandelt werden, so daß sie sozusagen einen „direkten Weg nach ganz EG-Europa” darstellen. Dieser neuen Konzeption wird wohl insbes. von seiten der britischen und der deutschen Regierung Sympathie entgegengebracht. Zugleich könnte dieses Modell aber auch eine strukturelle Wettbewerbsbeschränkung für alle übrigen Gesellschaftsformen bedeuten, so daß der Vortragende gegen eine derartige Europäische Gesellschaft erhebliche Bedenken hat, obwohl sie u. U. Konzernbildungen in Gestalt von Trauben nationaler Töchter überflüssig macht.

In diesem Zusammenhang muß gesagt werden, daß die Erhaltung der Marktstrukturen (große, mittlere und kleine Gesellschaften, AG, VVaG und öffentlich-rechtliche Versicherungsunternehmen) eine wesentlich größere Bedeutung hat, als bislang eingeräumt wurde. Für die Gegenseitigkeitsvereine ist übrigens, soweit ich unterrichtet bin, mit einer eingeschränkten Ausnahme in Frankreich, die Frage der Bildung von Konzernobergesellschaften nicht gelöst, sieht man von dem Gegenseitigkeitsverein selbst als Mutter von Aktiengesellschaften $\mathrm{ab}$. Im übrigen liegt die Vorstellung neben der Sache, daß große Risiken von großen, mittlere Risiken von mittleren und kleine Risiken von kleinen Gesellschaften zu decken seien. An dieser Frage sollten die Rückversicherer besonders interessiert sein. Es gehört in den Bereich reiner Theorie zu meinen, daß der Versicherungsbedarf am besten durch Versicherungsoligopole zu decken sei. Sie würden nur Vorläufer staatlicher Einheitsgesellschaften sein. Zur Niederlassungsfreiheit in diesem Zusammenhang noch eine letzte Bemerkung, die auch im Zeitalter der bereits genannten Deregulierung gewagt wird: Ein Vorteil des Niederlassungssystems besteht in der einfacheren Überwachbarkeit der Versicherungstätigkeiten nach einheitlichen Grundsätzen für In- und Ausländer und für alle Vertriebsformen. Das bedeutet teilweise auch Markttransparenz, Vergleichbarkeit der Preise mit Preiswettbewerb. Die Niederlassungsfreiheit kommt zudem dem Bedürfnis von Versicherten vieler, aber nicht aller Länder entgegen, sich bei einem örtlichen Versicherungsvertreter sicher zu fühlen. Aber die Niederlassungsfreiheit bedeutet keinen einheitlichen Markt mit breitem Modellwettbewerb.

3. Mit dem Paukenschlag, mit dem der EuGH in seinen vier Urteilen vom 04. 12. 86 der Beseitigung der Beschränkung der Dienstleistungsfreiheit neue Wege gewiesen hat, hatte die Praxis wohl kaum gerechnet, jedenfalls nicht, wenn es so ausgedrückt werden darf, mit einem ,praktikablen Paukenschlag”. Dabei war die These von der Natur der Sache nach unterschiedlicher Aufsichtsintensität für das Massen- und das Großgeschäft sogar im Rahmen der Interpretation des geltenden Aufsichtsrechts seit einer Reihe von Jahren vertreten worden. In den für das Mengen- und das Großgeschäft unterschiedlichen Regelungen 
kommt ein Kompromiß zwischen Konsumentenschutz im engeren Sinne, also „Konsumentenschutz durch Regulierung” und allgemeinem „Schutz des Versicherten durch Wettbewerb" zum Ausdruck. Dabei wird - entsprechend der Philosophie der Verwirklicher des Vertrages von Rom - dem Konsumentenschutz durch Regulierung nur eine gewisse Vorläufigkeit zuerkannt.

Über Schwellenwerte soll hier nicht erneut gesprochen werden, wohl aber muß angedeutet werden, daß die Einhaltung der Grenzen im praktischen Geschäft wohl nicht immer sicher ist, insbes. schwerlich von den Aufsichtsbehörden überwacht werden kann. Bereits hier wird das, wie ich es nennen möchte, Pluralismus-Problem der Dienstleistungsfreiheit deutlich. Der kreative Pluralismus wird bei wirklichen Übertreibungen zum Problem.

Aber damit ist nur ein kleines Stück der praktischen Probleme einer Tätigkeit zutage getreten, die Dr. Walter Diehl mit der Tätigkeit des „absentee-Versicherers” äußerst plastisch bezeichnet hat. Die so einflußreiche Wettbewerbstheorie verkennt nämlich, daß nicht etwa allein Werbung und Vertragsabschluß wettbewerbsrelevant sind, sondern vor allem die Erbringung der Versicherungsleistung. Die Konsumenten wissen ebenso wie die Großabnehmer von Versicherungsschutz, wie sich der Versicherer im Schaden- und im Ablauffall verhält, und beurteilen ihn danach. Gewiß ist das Regelungsmodell für die freien Dienstleistungsgeschäfte, das nach Einführung des Mehrheitsprinzips im Ministerrat verabschiedet worden ist, logisch bedeutsam. Ob es aber hinreichend kostengünstig ist, um attraktiv zu sein, und ob es für die Schadenregulierung hinreichend kundenfreundliche Regelungsmöglichkeiten offenläßt, bleibt dahingestellt. Dabei ist gewiß die Kritik überspitzt, die dahingeht, daß das Modell nur oder vor allem den Interessen der Versicherungsmakler entgegenkomme. Allerdings - das muß dialektisch eingewandt werden - ist der Makler im Denkmodell logisch notwendig, wenn man nicht einen in der Regel informationsfähigen und -bereiten Versicherungsnehmer fingieren will, der sowohl Preise als auch Produkte vergleichen kann. Über die Frage der Findungskosten geht man dann gern leicht hinweg. Auch Wirtschaftsmagazine füllen den Raum nicht etwa voll aus. Makler mit einer derartigen Kompetenz sind in hinreichender Zahl jedenfalls nicht in allen Mitgliedsländern tatsächlich vorhanden. Der weitergehende Konzentrationsvorgang im Maklerbereich bedarf in diesem Zusammenhang der Beobachtung. Die Erfahrungen mit im direct selling besonders auf dem Gebiet der Lebensversicherung tätigen Versicherungsunternehmen zeigen, daß, wie allgemein bekannt, die Märkte der auf diesem Gebiet ,urteilsfähigen” Versicherungsinteressenten $\mathbf{z}$. Z. nicht sehr groß sind. Zum Informationsproblem wird künftig noch mehr zu sagen sein.

Es war soeben von den Versicherungsmaklern die Rede, die im Modell als „Suchgehilfe" des Versicherungskonsumenten wesentliche Funktionen haben. In dem dichter werdenden Markt nimmt ohnehin der Absatz von Versicherungen - vom Unternehmen her Vertrieb genannt - einen noch wesentlicheren Platz als in der Vergangenheit ein. Die allerdings nur sporadisch vertretene - Ansicht, daß der Vertrieb als solcher wegen des höheren Angebots billiger werden würde, beruht gewiß auf einem Irrtum. Nach Ansicht des Referenten werden die Vertriebskosten wegen der Schlüsselstellung der Vertriebsorgane tendenziell eher steigen als fallen. Es ist zu wünschen, daß Mischformen zwischen den Maklern, den „Suchgehilfen” des Versicherungsnehmers, und den Agenten, den „Vertriebsgehilfen" des Versicherers, daß sog. Makleragenten noch seltener als heute anzutreffen sein werden. Sie sind bekanntlich nach dem Financial Services Act in Großbritannien 
widerrechtlich. Hierher gehören in einem weiteren Sinne auch Fälle, in denen Makler von Versicherern offen oder verdeckt beherrscht werden.

$\mathrm{Ob}$ besonders organisierte Prüfungen der beruflichen Kenntnis den Maklern als besonderer Vermittlergruppe nützen werden, lasse ich offen; die Erfahrungen in den Niederlanden sind offenbar positiv. In der Bundesrepublik bemüht man sich um ein System beruflicher Bildung allgemein von Versicherungsvermittlern. Bedenkt man den hohen Grad der Technisierung nicht nur der sog. technischen, sondern auch allgemein der Industrierisiken und zieht man die Bedeutung der Gefahrverhütung auch im Rahmen des Risk Management in Betracht, so bedeutet die Ausdehnung des Maklermarktes auch auf diesem Gebiet eine weitere Verdünnung des unmittelbaren Kontakts zwischen Versicherer und Versicherungsnehmer bei diesen Großrisiken. Es ist der Gedanke aufzugreifen, der hier eine Belastung sieht und anregt, nach noch wirksameren Wegen der Risikopflege zu suchen als in der Ebene des Underwriting. Es ist notwendig, auf dem Gebiet der Schadenvorsorge den Makler stärker zu engagieren; denn ihm fehlt das Eigeninteresse des Versicherungsnehmers, Schadenverhütungsinvestitionen durch finanzielle Vorsorge auf Zeit zu ersetzen, und dasjenige des Versicherers, die Aufwendungen im Schadenfalle möglichst niedrig zu halten.

Diese auf die Notwendigkeiten der Praxis abgestellten Überlegungen strahlen auf das sog. Kumulproblem aus. Das Kumulverbot bedeutet im Prinzip, daß derjenige EG-Versicherer, der eine auf Dauer angelegte Präsenz als faktische Niederlassung oder nach Art einer Agentur in einem Mitgliedsland unterhält, auch formell eine Niederlassung errichten muß. Dies gilt auch dann, wenn ein Büro nur von einer Person geführt wird, auch wenn diese unabhängig, aber beauftragt ist, für das Versicherungsunternehmen wie eine Agentur zu handeln. Wer überhaupt eine Niederlassung errichtet hat, muß sein Geschäft in dem betreffenden Land grundsätzlich über diese abwickeln. Ihm steht daneben Dienstleistungsfreiheit nicht zu (Kumul nicht erlaubt). Es sind unterschiedliche Strengegrade des Kumulverbots denkbar. Auf Ausnahmen kommt es hier nicht an. Es ist indessen nicht überzeugend, durch restriktive Auslegung des Begriffs Agentur zu das Kumulverbot abschwächenden Lösungen zu kommen. Die hier kritisierte These lautet: „Ein Agent ist noch nicht ohne weiteres eine Agentur". An dieser Stelle liegt sicherlich ein Schwächepunkt des gesamten Regelungsmodells, auch bezüglich der Einhaltung des Verbots. Das Kumulproblem, das aus der Konzeption des EuGH heraus in der Zweiten KoordRichtl Schaden behandelt worden ist, könnte u. a. auch im Zusammenhang mit der Harmonisierung der Pflichthaftpflichtversicherung der Kraftfahrzeughalter auf uns zukommen.

Ist ein Büro, das Schadenverhütung bezüglich der im Wege der freien Dienstleistung übernommenen Risiken betreibt, ein Tatbestand, der zur Niederlassungsnotwendigkeit führt bzw. die unzulässige Zweispurigkeit verkörpert? Dieselbe Frage stellt sich bezüglich der Schadenregulierungsbüros, die nach Richtlinien des Versicherers mehr oder weniger selbständig Schäden regulieren, ggf. auch Weisungen einholen. Sie sind bekanntlich in der Rechtsschutzversicherungsdirektive als ein legaler Weg zur Kollusionsverhütung sozusagen „EG-existent” geworden. Sind, so ist zu fragen, Schadenverhütungsbüros, die für mehr als einen Versicherer (mehr als eine Gruppe) tätig sind, anders zu beurteilen als Einfirmenregulierungsbüros? Aus deutschem Versicherungsaufsichtsrecht stellt sich zudem die Frage, ob es sich hier nicht um eine sog. Funktionsausgliederung, einen aufsichtsrechtlichen relevanten Tatbestand handelt, der die Schadenregulierung als essentielle Tätigkeit dem Versicherer zuordnet. Schließlich könnten bei von mehreren Versicherern betrauten 
Regulierungsbüros kartellrechtliche Fragen nach § 85 EWGV auftreten. Hier stellen sich zwei Fragen: Stellt eine Niederlassung eines Versicherungsmaklers, der als Suchgehilfe des Versicherungsnehmers vermittelt, aber eine Schadenregulierungsvollmacht des Versicherers innehat, eine Niederlassung des Versicherers dar? Wie ist der sog. technische Makler zu beurteilen, der auch noch in Vollmacht des Versicherers die Dokumente ausfertigt? Naturgemäß vertreten die Versicherungsmakler insofern die Auffassung, daß es sich nicht um Versicherungsniederlassungen handelt. Es bleiben Zweifel.

Für die Praxis wird es vor allem darauf ankommen, wie die Zweite KoordRichtl Schaden in die nationalen Rechte umgesetzt, insbes. wie die im Zusammenhang mit der DLF eingeräumten (wohl 20) Mitgliedstaatenwahlrechte ausgenutzt werden.

4. Koordinierung - hier der Dienstleistungsmöglichkeiten - und Deregulierung, also Liberalisierung, gehören zu den wesentlichen Zielen des Vertrages von Rom. Sowohl nach seinem Denkmodell als auch unter rein praktischen Gesichtspunkten haben Liberalisierung und Rückzug des Staates aus den juristischen Rahmenbedingungen wirtschaftlicher Tätigkeit stets zur Folge, daß die Wettbewerbsbedingungen überprüft und normalerweise verschärft werden. Die Substitution staatlicher Regulierung durch Kartelle soll verhindert, den etwaigen Ausweitungen des unlauteren Wettbewerbs entgegengewirkt werden. Das, was den guten Sitten entspricht, pflegt enger umgrenzt zu werden.

Genau dies vollzieht sich in dem jetzigen Entwicklungsabschnitt des Gemeinsamen Marktes, wobei man - wie erwähnt - auch hier nicht auf 1992 wartet. Für das Kartellrecht, das nach einer Entscheidung des EuGH vom Februar 1987 auch auf Unternehmen mit Sitz in einem Nicht-Mitgliedstaat anwendbar ist, ergibt sich ein besonders ausgeprägtes Problem des Verhältnisses von EG-Recht zum nationalen Recht. Sie hören hier keine Vorlesung über Versicherungskartellrecht. Es muß aber auf die Interdependenz zwischen Regulierung von AVB, also Genehmigung und Überwachung, einerseits und der kartellrechtlichen Beurteilung von Versicherungsbedingungen hingewiesen werden. Von den ökonomischen Wettbewerbstheoretikern wird dazu mit großem Nachdruck die Meinung vertreten, daß die Schutzfunktion staatlich fixierter Rahmenbedingungen sehr weitgehend durch den Wettbewerb übernommen werde. Auch hier geht deshalb die herrschende Meinung dahin, den Freiraum, der durch Regulierung entsteht, nicht in die „Hände von Kartellen fallen zu lassen”, wenn ich dies so ausdrücken darf. Auch hier bezweifeln alte Praktiker, daß die selbstheilenden Kräfte des Marktes bis in die Winkel des sog. Kleingedruckten reichen oder daß die Makler eine Schutzfunktion bezüglich der AVB im Mengengeschäft im Interesse der Konsumenten ausüben werden. Deshalb wird dafür plädiert, die in der Zweiten KoordDir für das Massengeschäft eröffneten Möglichkeiten der Aufrechterhaltung bzw. Neueinführung der Bedingungsgenehmigung auszuschöpfen. Zu den Gruppenfreistellungswünschen zum EG-Kartellrecht gehören neben der Vertragsrückund -mitversicherung insbesondere auch die AVB. Die Schutzsysteme der Mitgliedstaaten sind bezüglich der AVB nicht äquivalent. Soweit Rechtswahlfreiheit eingeräumt ist, können sie zudem umgangen werden. Zum Verhältnis zwischen EG-Kartellrecht und nationalen Vorschriften gegen Wettbewerbsbeschränkungen genügt es, hier darauf hinzuweisen, daß es um die Frage eines absoluten oder nur relativen Vorrangs des EG-Rechts geht. Die inneren Teilmärkte des Gemeinsamen Binnenmarkts sollen offenbar weiterhin unter nationalen Kartellrechten leben, deren Verschiedenheit gewiß zu den mehr oder weniger sichtbaren Handelshemmnissen gehören wird. 
Unter den rechtlichen Regelungen des Wettbewerbs nimmt die sog. Fusionskontrolle einen besonderen Platz ein. Unternehmenszusammenschlüsse stoßen unter dem Gesichtspunkt der Eignung zur Marktbeherrschung im Falle, daß eine solche ausgeübt wird, auf gesetzliche Schranken. Dabei wird im Regelungsmodell grundsätzlich eine staatliche Kontrolle ex ante, also das Erfordernis staatlicher Genehmigung, in möglicherweise ganz verschiedenartiger Ausgestaltung vorgesehen. Wenn man es so sehen will, handelt es sich um eine Marktzutrittsschranke für Großunternehmen im Falle der Entstehung durch Rechtsgeschäft, also um eine „Höherverlegung” der Konzession. Weil Großunternehmen einerseits Instrumente der einzelstaatlichen Industriepolitik (d. h. der interventionistischen Politik zur Förderung der Volkswirtschaft) sein können, andererseits aber unter dem Gesichtspunkt der Wettbewerbsbeschränkung im großen Binnenmarkt auf Kritik stoßen, kommt der Beurteilung des Gemeininteresses hier große Bedeutung zu.

Es drängt sich außerdem die Feststellung auf, daß „kritische Größe” im nationalen Teilmarkt, im EG-Binnenmarkt und gar im Weltmarkt verschieden zu dimensionieren ist. Die kommende EG-Regelung stellt nicht nur eine höchst interessante Aufgabe, bedeutet aber auch eine gesetzgeberische Entscheidung von größter Tragweite für die Unternehmen und damit auch für die Volkswirtschaften der Mitgliedstaaten. Denn hierher gehört nicht nur die Feststellung, daß der wirtschaftende Staat selbst Großunternehmen unterhält und in jüngerer Zeit solche geschaffen hat, sondern daß der Mechanismus von Unternehmenskäufen (friendly und unfriendly take-overs) die betonte Frage stellt, wo Freiheit über die bei allen Unternehmensübernahmen zu prüfende Marktbeherrschungsfrage hinaus durch den individuellen Übernahmevorgang mißbraucht wird. Noch ein letzte Satz zu der Wettbewerbsordnung, die gemeinschaftsrechtlich begründet sein muß, wenn es einen gemeinsamen Markt gibt: der Dumping-Begriff ist bisher für Versicherungsgeschäfte kaum befriedigend definiert worden. Wo liegt die Grenze des Erlaubten, wenn hohe Verluste eingeplant sind, um durch Unterbietung in den Markt einzudringen?

In engem Zusammenhang mit dem soeben erörterten Komplex steht die jedenfalls für das Großgeschäft eingeräumte bereits erwähnte Rechtswahlfreiheit, d. h. die den Vertragsparteien des einzelnen Versicherungsvertrages eingeräumte Möglichkeit, die anzuwendende nationale Rechtsordnung frei zu wählen. Es wird also der im Internationalen Privatrecht (dem Kollisionsrecht) entwickelte Gedanke konkretisiert, daß primär der Parteiwille darüber entscheidet, nach welcher Rechtsordnung sich ein Vertrag richtet. Es ist nicht leicht vorauszusagen, wie sich die Praxis entwickeln wird. Vor allem die Umgrenzung des nicht abdingbaren zwingenden nationalen Rechts wird schwierig. Auf die konkreten Regelungen werden der Makler und auch der Versicherer Einfluß ausüben. Die Einräumung der Rechtswahlfreiheit stellt aber einen in erster Linie von der Logik her motivierten Behelf dar, trotz fehlender Koordinierung des Versicherungsvertragsrechts, Diskriminierungen zu vermeiden. Auch hier kritisiere ich den geringen Koordinierungsgrad nicht, sondern stelle ihn nur dar. Es gilt eben der Satz „volenti non fit in iuria”, hier konkret: „Wer sich selbst die Rechtsordnung ausgesucht hat, ist nich diskriminiert".

Erlauben Sie mir an dieser Stelle einen kurzen Exkurs: Es ist bekannt, daß das zusätzliche Rechtsverwirklichungsrisiko dann sehr groß ist, wenn man eine fremde Rechtsordnung wählt. Das normale Risiko ist wesentlich erhöht. Deshalb werden m. E. zukünftig Schiedsgerichtsabreden, also vertragliche Vereinbarungen, daß ein etwaiger Rechtsstreit durch ein Schiedsgericht zu entscheiden ist, auch im Bereich der Leistungsklagen gegen 
Versicherer besondere Bedeutung haben. Es gibt erprobte Verfahren und Verfahrenshilfen, an die man denken sollte, also Schiedsgerichtsverfahren über die gängigen Schiedsgutachterverfahren hinaus.

5. Es fehlt in diesem Gesamtgedankengang noch ein wesentliches Element. Wie Sie wissen, liegt der Ansatzpunkt für die Schaffung der Versicherungsaufsicht in dem Gedanken, den Versicherungsnehmer als Gläubiger der häufig langfristig zu erbringenden Versicherungsleistungen zu schützen, mögen auch die Aufsichtsbehörden den Schuldnerschutzgedanken vor allem unter Einfluß der Konsumentenschutzbewegung hinzugenommen haben. Fällt der Gläubigerschutz durch Deregulierung aus oder wird er gemindert, stellt sich die Frage nach einem „Aufsichtsersatz”. Nachdem man Überlegungen zur Pflichtrückversicherung als nicht in einen freiheitlichen Markt passend mit Recht aufgegeben hat, glaubt man, ihn in Konkurssicherungsfonds zu finden, also in der kollektiven Garantie der Branche bzw. von Teilbranchen. Solche Fonds, die es bekanntlich besonders in den USA gibt, sind in der Versicherungswirtschaft heftig umstritten, weil die „Ordentlichen” nicht nur für die „Unglücklichen”, sondern vor allem auch für die „Unordentlichen” einstehen müßten. In diesem Zusammenhang ergeben sich Fragen zum Einfluß der Fonds auf das Geschäftsgebaren, also zu Überwachungskompetenzen der Fonds. Sie ersetzen dann nicht nur Schutz-, sondern auch Interventionsfunktionen des Staates.

6. Es bleiben noch abrundende Bemerkungen in diesem Vortrag, der absichtlich nicht mit Vorschlägen in die Rechtsetzungsvorgänge eingreift, die in vollem Gange sind. Auch eine Gesamtdarstellung der juristischen Probleme würde viel zu weit gehen. Es geht mir, wie erwähnt, um die Bedeutung der Regelungen für das praktische Versicherungsgeschäft.

Dazu gehört vor allem auch die Lebensversicherung, für welche eine Zweite KoordRichtl in systematischer Anlehnung an die entsprechende Schadenversicherungsdirektive vorbereitet wird. Man überlegt, ob und ggf. in welchen Bereichen das Sitzlandsprinzip, also die Beaufsichtigung durch die Behörde des Sitzlandes, verwirklicht werden kann. Eine einfache Unterscheidung zwischen Mengen- und Großgeschäft bietet sich für die Lebensversicherung nicht an. Es ist sicherlich allein nicht richtig, hier von einer Aussonderung von sog. Großgeschäft, das man dem Sitzlandprinzip unterwerfen würde, abzusehen. Schwellenwerte sind hier nämlich als Kriterium ungeeignet, wie keiner Begründung bedarf. Aber auch Gruppenversicherungen reichen nicht aus dem für Massenrisiken kennzeichnenden Schutzbereich heraus; denn es sind in der weitaus überwiegenden Zahl der Fälle aus Rationalisierungsgründen zusammengefaßte Massenrisiken, bei denen die Versicherten schutzbedürftig sind. Auch ergeben sich Fragen zum Niederlassungsbegriff hinsichtlich der Gruppenspitze. Es ist interessant, daß z. B. durch Makler im Bereich der Gruppenversicherung durch Anzeigen geworben wird. Auf die ungelöste Steuerharmonisierung muß hier erneut hingewiesen werden.

Von erheblicher Bedeutung für die Lebensversicherung ist die Kapitalverkehrsrichtlinie vom Juni 1988, durch die Beschränkungen des Kapitalverkehrs beseitigt werden. Dabei wurde der Transfer von Prämien und Leistungen der Lebensversicherung förmlich liberalisiert. Von Bedeutung ist der Definitionenkatalog, die sog. Nomenklatur des Kapitalverkehrs, die der Direktive beigegeben ist.

Damit ist aber hier auch das Stichwort „kombinierte Finanzdienstleistungen” gefallen, über das in unseren Ländern viel geredet und geschrieben worden ist. In Anlehnung an 
Pierre Moussa, der kürzlich vor dem CEA gesprochen hat, meine ich, daß die Beherrschung von Versicherungsunternehmen durch Banken und umgekehrt diejenige von Banken durch Versicherungen vermieden werden sollte. Die Konstruktion einer Finanzholding über beiden erscheint attraktiver. Die Kombination der Vertriebswege und die Schaffung entsprechender Produkte spielen eine große Rolle.

Am Schluß dieses Vortrags fragen Sie mich vielleicht, wie ich die EG-Teilmärkte nach Durchführung der laufenden Rechtsetzungsmaßnahmen beurteile. Wird es praktische Dienstleistungsgeschäfte ohne nationale Niederlassungen in nennenswertem Umfang geben? Ich meine zunächst, daß für das bekanntlich in zwei Stufen behandelte Großgeschäft diese Vertriebsart eine der normalen sein wird. Es könnte allerdings sein, daß sich die Regelungen im einzelnen als zu kompliziert erweisen, so daß die Kunden verschreckt sein könnten. Andererseits könnten die Kunden, die zunächst den Flötentönen des reinen Dienstleistungsverkehrs folgen, nach Ablauf einer gewissen Zeit zurückkehren. Die Entwicklung hängt von Größe der Risiken, der praktischen Möglichkeit für Industriekonzerne, wirklich eine transparente Generaldeckung auf DLF-Basis zu haben, von Preisgestaltungen, Regulierungsverhalten der Versicherer und auch Funktionen der Makler ab. Insoweit aber meines Erachtens: Bewegung, aber keine grundstürzenden Besonderheiten.

Anders beurteile ich das Massengeschäft. Wenn man hier den Gedanken des reinen Dienstleistungsgeschäfts überspannt, schädigt man die Vertrauensbasis des Versicherungsgeschäfts überhaupt. „Lieschen Müller und Klein-Erna” werden sich meines Erachtens im Ergebnis bei dem präsenten Versicherer einfach wohler fühlen. In diesem Zusammenhang spielt die genannte AVB-Frage eine große Rolle, ebenso wie das praktische Verhalten der Gerichte. Doch sind solche Prognosen äußerst unsicher, vor allem, soweit sie über die unmittelbar vor uns liegenden Jahre hinausgehen. Und im Gegensatz zur theoretischen Ökonomie, wo man von dem rational handelnden Verbraucher ausgeht, sind nach der Massenpsychologie im Massenmarkt Irrationalitäten normal.

Schließlich muß ich der Ordnung halber noch dem Komplex der sog. Korrespondenzverträge anschneiden. Hier handelt es sich aber nicht nur um briefliche oder durch elektrische oder elektronische Medien aus alleiniger Initiative des Versicherungsinteressenten übermittelte Offerten, sondern vor allem auch um persönliche Abschlüsse bei Aufenthalt des Interessenten außerhalb seines Hoheitsgebiets. Die These geht dahin, daß demjenigen, der sich willentlich des Schutzes seines Staates in der gekennzeichneten Weise begibt, ein solcher Schutz auch nicht gewährt wird. Es gibt eine ganze Menge von ergänzenden Fragen: So gibt es z. B. zur Zeit, soweit ich sehe, Korrespondenzversicherung in der EG nur im deutschen Aufsichtsrecht in einem engeren Tatbestand. Wie, so ist weiter zu fragen, sind Versicherungsverträge zu beurteilen, die Dritten, die sich ihrerseits nicht aus dem Schutzbereich ihres Gesetzgebers herausbegeben haben, eigene Rechtsansprüche gegen den Versicherer gewähren? Kann der Versicherungsnehmer bei Abschluß ihre Rechtsposition „mindern”? Die Bestimmungen der nationalen Rechte, die Versicherungspflichten begründen - es gibt sehr zahlreiche -, schließen wohl Korrespondenzverträge aus. Es wird schließlich die wesentliche Meinung vertreten, daß dort keine Korrespondenzversicherung zulässig ist, wo der Abschluß auf Grund der Werbung durch den Versicherer oder unter Mitwirkung nationaler Vermittler zustande gekommen ist.

Die Entwicklung des Gemeinsamen Binnenmarktes geht jetzt offenbar mit recht schnellen Schritten voran. Bezüglich der künftigen Entwicklung kann man die Behandlung des Großgeschäfts in etwa voraussagen. Es wird ein etwas größerer Teil der nationalen 
Industrieversicherungsportefeuilles in andere Mitgliedsländer gehen. Darüber wird es Pendelbewegungen geben. Man kann davon ausgehen, daß die als Industrieversicherer renommierten Versicherungsunternehmen weiterhin auch beträchtliche nationale Bestände haben werden. Nach meiner Vorstellung wird der Satz gelten: „Der niedergelassene Versicherer hat ein Prä vor dem absentee”. Bezüglich des Mengengeschäfts wird hoffentlich nicht erst durch Zusammenbrüche und sonstige Skandale die Überzeugung wieder mehr Gewicht haben, daß vor allem überall dort, wo Dritte durch Privatversicherungsverträge mitbetroffen sind, das Liberalisierungsinteresse des Versicherungsnehmers gegenüber den Interessen der Allgemeinheit zurücktreten muß. Denken Sie nicht nur an die Pflichtversicherungen, sondern auch an die Bezugsberechtigten bei der Lebens- und Unfallversicherung und an die übrigen Verträge mit Schutzwirkungen zugunsten Dritter. Man könnte sich vorstellen, daß sich die wesentlichsten Auswirkungen bezüglich der Unternehmensgrößen, -struktur und Gruppenbildung ergeben. Dieser Vortrag, der im Rahmen der Genfer Vereinigung gehalten wird, steht im Dienste eines sachlichen und aufgeschlossenen nachbarlichen Gesprächs über eine Sache, die dem Vortragenden und ganz offenbar auch den Hörern am Herzen liegt. 